\title{
A TECHNIQUE FOR COMBINING EQUALIZATION WITH GENERALIZED DIFFERENTIAL DETECTION
}

\author{
by : \\ K. M. Aleong* , H. Leib, P. Kabal \\ Dept. of Electrical Eng., McGill University \\ Montreal, Que, H3A 2A7, CANADA
}

\begin{abstract}
This work considers a generalized differential coherent detection technique combined with equalization for data transmission over intersymbol interference (ISI) channels when carrier phase tracking is difficult. In the absence of ISI, the SNR performance of this generalized differential coherent structure is very close to the coherent detection bound. This paper presents the integration of this generalized differential detection method with channel equalization, and therefore it extends the applications of this detection technique to channels with ISI. It is shown that for high SNR, the performance degradation of this structure with respect to coherent detection and equalization is negligible. Analysis and computer simulations show that this combined detection/equalization technique is an effective solution to the ISI problem when carrier phase tracking is difficult. One possible application of this work is in indoor wireless communications.
\end{abstract}

\section{INTRODUCTION}

Due to the increased interest in communication over channels where carrier-phase-tracking is difficult (such as fading multipath channels, TDMA systems, Frequency Hopping), new differentially coherent demodulation techniques have been introduced in the last few years [1]-[4]. A feature of these detection methods is their low SNR degradation with respect to corresponding coherent detectors. However, many of the systems that these differentially coherent techniques aim at, are also impaired by intersymbol interference (ISI). Therefore, the extent to which these new differentially coherent detection techniques can be applied in practice depends on the possibility of combining them with equalization.

We consider the generalized differential detection method introduced in [1]. This structure can be naturally combined with known equalization techniques, while the other proposed differentially coherent detectors, [2]-[4] seem to require special equalization methods. In this work we consider linear equalization to demonstrate the technique of integration with differential detection. This concept is exten- sible to decision-feedback equalization as well. The present work considers bandwidth efficient two dimensional modulation, where the data is encoded into the phase and amplitude of the carrier. In order to avoid carrier phase tracking, we use differential phase encoding and therefore differential coherent detection techniques can be employed.

The paper is organized along the following lines. Section 2 introduces the system model. It presents the generalized differential coherent detection technique and its integration with equalization. Section 3 analyzes the minimum mean square error which can be obtained with this structure. Section 4 considers adaptive equalization with generalized dif ferential detection, and presents simulation results for some multipath channels. Finally, section 5 presents the summary and conclusions of this work.

\section{INTEGRATION OF EQUALIZATION WITH GENERALIZED DIFFERENTIAL COHERENT DETECTION}

Two dimensional modulation where the data is encoded into the phase and amplitude of the carrier has been extensively employed for bandwidth efficient digital transmission [5]. A close relative to this modulation format is amplitude and differential phase modulation. Differential phase modulation structures the carrier such that phase differences and not absolute carrier phases convey information. Therefore with differential phase modulation, absolute carrier phase tracking is not necessary since the receiver can employ differentially coherent detection.

A differentially coherent detector estimates the transmitted data by making use of phase differences between successive symbols. The SNR degradation of differential detection with respect to corresponding coherent detection depends on the specific signaling format. For Binary Phase Shift Keying (BPSK) this degradation is less than one $\mathrm{dB}$; however for larger signal constellations, this degradation approaches 3 dB. Many techniques have been proposed for decreasing this SNR degradation without the need of carrier phase tracking (see [1]-[4] and the corresponding references). We focus here on a particular technique introduced in [1], and this work considers its applica-

This work was supported by grants from the Natural Sciences and Engineering Research Council (NSERC) of Canada.

* This author is now with MacDonald Dettweiler and Associates, Vancouver BC Canada. 
tion for ISI channels.

An ideal coherent system uses a noise free phase reference and this is the reason for the improved SNR performance. With differential phase coding, the reference phase required for data detection is carried by the previous symbol. This reference is impaired by the channel noise in the same manner as the information conveying symbol. The receiver introduced in [1] creates a phase reference by employing $L$ past symbols. Therefore it smoothes the effects of the channel noise, and by doing so it increases the SNR of the phase reference. It has been shown in [1] that without ISI, as $L \rightarrow \infty$ the performance of the generalized differential detector tends to the coherent detection bound. On the other hand when $L=1$ the structure degenerates to usual differential coherent detection.

The baseband model (complex envelope (CE)) for the system considered in this work is illustrated in Fig.1. The transmitter is modeled by a differential phase encoder followed by a shaping filter $\tilde{g}_{T}(t)$. The $\mathrm{CE}$ of the transmitted signal is given by $\tilde{s}(t)=\sum_{k=0}^{\infty} b[k] e^{j \phi[k]} \tilde{g}_{T}(t-k T)$, where $b[k] e^{j \phi[k]}$ are the amplitude and differentially phase-encoded symbols transmitted at a rate of $1 / T$ [symbols/sec ]. The transmitted amplitude and differentially encoded phase in each symbol interval are related to the two dimensional constellation points in an obvious way. The transmitted amplitude $b[k]$ is identical to the amplitude of the constellation point while the transmitted phase $\phi[k]$ is a differentially encoded version of the constellation point phase $\psi[k]$; thus $\phi[k]=\phi[k-1]+\psi[k]$. In this work we consider the two dimensional constellations illustrated in Fig.2 : QPSK, 8PSK, 16QAM, 8V29 and 16V29. The 8V29 constellation consists of 8 inner points of the standard $16 \mathrm{~V} 29$ constellation. Average power of each constellation has been normalized to unity.

We assume a linear, additive Gaussian channel. With multipath, the $\mathrm{CE}$ of the channel impulse response is $\tilde{g}_{C}(t)=\sum_{i=1}^{p} \rho[i] e^{j \theta[i]} \delta(t-\tau[i])$, where $N_{p}$ is the number of specular paths and $\rho[i], \theta[i], \tau[i]$ are the gain, phase and delay in the $i-t h$ path. The CE of the channel noise, $\tilde{n}(t)$, is a white zero mean complex Gaussian process with power spectral density of $N_{o}$ [Watts/Hz] in each of the real and imaginary components which are independent. Therefore, the CE of the received signal can be expressed as $\tilde{r}(t)=\tilde{s}(t)^{*} \tilde{g}_{c}(t)+\tilde{n}(t)$, where $*$ denotes the convolution operator.

The front end filter of the receiver has an impulse response $\tilde{g}_{R}(t)$. The receiver and transmitter filters, $\tilde{g}_{T}(t)$ and $\tilde{g}_{R}(t)$, are designed such that the overall response $\bar{g}_{T}(t)^{*} \bar{g}_{R}(t)$ satisfies Nyquist's first criterion. In this work we assume zero excess bandwidth and identical filters at receiver and transmitter. Therefore the frequency responses of these

$$
\text { filters }
$$

$\tilde{G}_{R}(f)=\tilde{G}_{T}(f)=\left\{\begin{array}{ll}T, & |f| \leq \mathcal{V}(2 T) \\ 0, & \text { else }\end{array}\right.$. The CE of receiver filter output can be expressed as :

$\tilde{y}(t)=\sum_{k=-\infty}^{\infty} b[k] e^{j \phi[k]} \tilde{g}(t-k T)+\tilde{n}_{R}(t)$

where $\tilde{g}(t)=\tilde{g}_{T}(t)^{*} \tilde{g}_{C}(t)^{*} \tilde{g}_{R}(t)$ is the overall continuous time response of the transmitter channel and receiver, and $\tilde{n}_{R}(t)=\int_{-\infty}^{\infty} \tilde{n}(t-\tau) \tilde{g}_{R}(\tau) d \tau . \quad$ The receiver filter output is sampled at the symbol rate, and these samples $\tilde{y}(n T)=y[n]$ are processed by the equalizer and used in the detection process.

The sampled signal at the input of the equalizer is

$y[n]=\sum_{k=-\infty}^{\infty} b[k] e^{j \phi[k]} g[n-k]+n_{R}[n]$

where $g[n-k]=\tilde{g}([n-k] T)$ and $n_{R}[n]=\tilde{n}_{R}(n T)$. In this work we consider a complex linear equalizer. The analysis can be generalized to a decisionfeedback equalizer as well. The linear equalizer consists of $2 N+1$ complex taps; the coefficients at the $n$-th symbol interval are $c_{k}[n], k_{N}=-N, \cdots, 0, \cdots, N$, and the output is $z[n]=\sum_{k=-N}^{N} c_{k}[n] y[n-k]$.

Following the technique of [1], the differential coherent detector utilizes $z[n-1], z[n-2], \cdots, z[n-L]$ to create a phase reference. First, all these samples must be rotated so they will be aligned with $z[n-1]$ and then the phase reference is obtained by summing the aligned samples. This phase references, $\hat{\beta}[n]$ is given by :

$$
\begin{aligned}
v[n] & =|v[n]| e^{j \beta[n]} \\
& =\sum_{i=1}^{L} z[n-i] \exp \left(j \sum_{k=1}^{i-1} \psi[n-k]\right)
\end{aligned}
$$

It is seen that the information bearing phases $\psi[n-1], \cdots, \psi[n-L]$ are used in (3). A training sequence is used for initial acquisition. In normal operation the receiver must use the previous decisions $\hat{\psi}[n-1], \cdots \hat{\psi}[n-L]$ ( decision-directed mode). The reference sample $v[n]$ is a result of a coherent summation which tends to increase the SNR; thus, its phase $\hat{\beta}[n]$ is a better estimate of the exact phase reference $\phi[n-1]$ than just the phase of the prevjous sample $z[n-1]$. The decision variable is $z[n] e^{-j \beta[n]}$. The decision is in the favor of the constellation point $\hat{b} e^{j \psi}$ which minimizes the magnitude of the error

$\varepsilon[n]=b[n] e^{j \psi \mid n]}-z[n] e^{-j \beta x[n]}$

In an adaptive mode of operation, this error is also used to adapt the equalizer coefficients. The analysis of this structure is presented in the next section. Simulation results for adaptive operation are presented in section 4 .

\section{MINIMUM MEAN-SQUARE ERROR ANALYSIS}

The mean-square error (MSE) is given by $E\left\{|\varepsilon[n]|^{2}\right\}$, where $\varepsilon[n]$ is the error defined in (4). 
It is assumed that the data symbols $b[n] e^{j \psi[n]}$ are statistically independent and normalized such that $E\left\{b^{2}[n]\right\}=1$.

The coefficients of the equalizer which minimizes the MSE are given by

$\underline{C}_{\text {opt }}[n]=A^{-1} \underline{\hat{B}}$

where $A$ is the input autocorrelation matrix defined by

$A=E\left\{y^{*}[n] y^{T}[n]\right\}$

$\hat{B}$ is the crosscorrelation vector defined by

$\hat{\hat{B}}=E\left\{b[n] e^{j \psi[n]} y^{*}[n] e^{j \hat{\beta}[n]}\right\}$

and $y[n]=[y[n-N], \cdots, y[n], \cdots, y[n+N]]^{T}$. The minimum MSE (MMSE) associated with this optimal equalizer is given by :

$\xi_{\min }=1-\underline{B}^{T^{*}} A^{-1} \underline{\hat{B}}$

From (5) we see that the crosscorrelation vector $\hat{B}$ depends on the reference phase estimate $\hat{\beta}[n]$ which is related to the exact phase reference $\phi(n-1)$ via

$\hat{\beta}[n]=\phi(n-1)+\eta[n]$

where $\eta[n]$ is the estimation error. From (3) we see that the estimate $\beta[n]$ is formed from $L$ samples of the equalizer output $z[n-1], \cdots, z[n-L]$, and each such sample $z[n-i]$ is a linear combination of the components of $y[n-i]$. Employing the well known independence assumption used in the theory of adaptive equalization, [6], we have that $\{y[n-i], i=0,1, \cdots\}$ can be considered statistically independent random vectors. This gives that $\{z[n-i], i=1,2, \cdots\}$ can be considered independent of $y[n]$ and therefore the estimate $\beta[n]$ is independent of $y[n]$. This is equivalent to the estimation error $\eta[n]$ being independent of $y[n]$. Under these circumstances we have :

$\hat{B}=\underline{B} E\left[e^{j \eta[n]}\right]$

where $B$ is the crosscorrelation vector with perfect reference phase estimate, i.e. $B=E\left[b[n] e^{j \phi[n]} y^{*}[n]\right]$. The term $E\left[e^{j \eta[n]}\right]$ is the characteristic function of the estimation error $\eta[n]$. Therefore $E\left[e^{j \eta[n]}\right]<1$ which with (6) shows that the reference phase estimation error increases the MMSE. For an unbiased reference phase estimator with a small error we can use the approximation $E\left[e^{j \eta[n]}\right] \approx 1-(1 / 2) E\left[\eta^{2}[n]\right]$, and in [7] it has been shown that the variance of the reference phase error can be approximated by :

$E\left[\eta^{2}[n]\right] \approx$

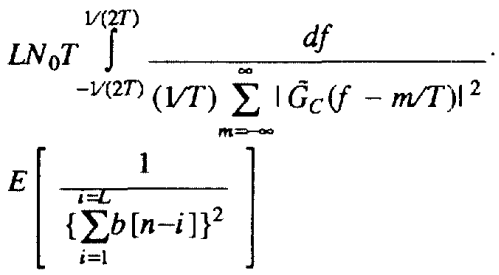

From (9) we see that the number of symbols used in the generalized differential coherent receiver affects the reference phase error variance via the term $H=L \cdot E\left[\frac{1}{\left\{\sum_{i=1}^{L} b[n-i]\right\}^{2}}\right]$

For MPSK $b[n-i]=1$ and thus $H=V L$ showing that the variance of the reference error is inverse proportional to $L$, and thus it can be reduced by increasing $L$. This holds also for amplitude and phase signal constellations since $b[n-i]>0$, and thus also in this case $H$ decreases with $L$. Therefore if the reference symbol $v[n]$ is smoothed over a sufficiently large number of symbols, the reference phase perturbation becomes insignificant, and the overall performance tends to the coherent detection bound for an equalized signal. It has been found that increasing $L$ from 1 to 2 results in a significant reduction in MMSE; however for $L>5$, the reduction in MMSE is small since the performance is already close to the coherent detection bound for an equalized signal. It appears that $L=3$ offers a good trade-off between reduction of the MMSE and memory in the generalized differential coherent receiver. Furthermore, the gain in going from $L=1$ to $L=2$ for amplitude/phase constellations is larger than for comparable MPSK constellations. Next we investigate this structure with an adaptive mode of operation.

\section{ADAPTIVE EQUALIZATION COMBINED WITH GENERALIZED DIFFERENTIAL COHERENT DETECTION}

In this section we consider the combined generalized differential detector and equalizer of Fig.1. when the coefficients of the equalizer are adapted to minimize the MSE. The adaptation algorithm considered in this paper is the LMS [8]. If necessary, algorithms with faster convergence, such as those based on Kalman filtering [9], can be used. With the LMS algorithm, the equalizer coefficient vector $c[n]$ is updated according to

$\underline{c}[n+1]=\underline{c}[n]+\lambda \varepsilon[n] y^{*}[n] e^{j \beta[n]}$

where $\varepsilon[n]=b[n] e^{j \phi[n]}-z[n] e^{-j \beta[n]}$ is the error at the $n$-th iteration and $b[n] e^{j \phi[n]}$ is a differential phase encoded symbol of the training data.

The operation of the system over two multipath channels with additive Gaussian noise was simulated on a computer. The two multipath channels that were considered are characterized by the following $C E$ impulse responses :

Channel $A: \tilde{g}_{C}(t)=\delta(t)-0.5 \delta(t-0.5 T)$

Channel $X: \tilde{g}_{C}(t)=\delta(t)-0.3 \delta(t-0.5 T)+$

$$
0.5 j \delta(t-3.5 T)
$$

where $T$ is the symbol time. An equalizer length $(N)$ of 9 was used. The number of symbols used in the differential coherent receiver for forming the reference phase $(L)$ was fixed to 3 . Each simulation used a randomly chosen training sequence (known to the 
receiver) of length 3220 symbols. All the equalizer coefficients were initialized to zero for each trial. The simulations were performed at an SNR of $25 \mathrm{~dB}$. It was found that sixty trials are needed to obtain reasonable smooth average learning curves. Fig.3 presents the results of sixty trials performed for 16QAM over the channel $X$. An LMS step size of 0.005 was used. It is seen that the average learning curve is reasonable smooth. Similar results were obtained also for other constellations over the two channels $A$ and $X$.

Simulations were performed for the four constellations 8PSK, 8V29, 16V29 and 16QAM. For the sake of brevity we present in this paper only the results for $16 \mathrm{QAM}$ and $16 \mathrm{~V} 29$. Two step sizes were used , $\lambda=0.05,0.005$. Figures 4 and 5 present the average learning curve for 16QAM and $16 \mathrm{~V} 29$ respectively. Notice that these two figures use a logarithmic scale for the MSE. From Fig.4 and Fig.5 it is seen that the larger step size resulted in a much faster convergence, typically within 200 iterations for the two channels and the two signal constellations. However the residual MSE is larger. A step size of 0.005 resulted in a slower convergence, typically within 1500 iterations for the two constellations over channel $A$ and 900 iterations over channel $X$. However this smaller step size yields a reduced residual MSE. Table 1 compares the MMSE with the residual MSE for the four constellations over the two channels with the two step sizes. It is seen that the smaller step size allows the residual MSE to be close to the MMSE. Furthermore, the residual MSE for the different constellations over the two channels is very close to each other, however the MMSE for channel $\mathrm{X}$ is lower than that of channel $A$

\section{SUMMARY AND CONCLUSIONS}

A combined equalization and generalized differentially coherent detection technique was presented. This structure is particularly suited for dispersive channels when traditional carrier phase tracking cannot be employed. In this work we aimed at bandwidth efficient indoor communications systems. Such systems are impaired by multipath and fading. A receiver which can cope with these impairments and can afford not to use carrier phase tracking without reducing the SNR efficiency, seems to be of special interest.

This work shows that the combination of standard equalization with the generalized differential detection technique of [1] results in a structure which can cope with intersymbol interference without the need of carrier phase tracking. The LMS algorithm was employed to demonstrate the feasibility of using this technique in an adaptive mode. If necessary, faster convergence algorithms, such as those based on Kalman filtering, could be employed.

\section{REFERENCES}

[1] H. Leib, S. Pasupathy, "The Phase of a Vector Perturbed by Gaussian Noise and Differentially
Coherent Receivers", IEEE Trans. Inform Theory, vol. 34, no. 6, pp. 1491-1501, Nov 1988.

[2] S. G. Wilson, J. Freebersyser, C. Marshall, "Multisymbol Detection of DPSK", GLO BECOM Conf., Dallas, Texas, pp. 1692-1697, Nov. 27-30, 1989.

[3] D. Divsalar, M. K. Simon, "Multiple-Symbol Differential Detection of MPSK", IEEE Trans. Comm., vol. COM-38, no. 3, pp. 300-308, Mar. 1990.

[4] H. Leib, S. Pasupathy, "Optimal Noncoherent Block Demodulation of Differential Phase Shift Keying (DPSK)", AEU International Journal of Electr. and Comm., vol. 45, no. 5, pp. 299. 305.

[5] G. D. Fomey Jr., R. G. Gallager, G. R. Lang, F. M. Longstaff, S. U. Qureshi, "Efficien Modulation for Band-limited Channels", IEEE J. Sel. Areas Comm., vol. SAC-2, no. 5, pp. 632-647, Sept. 1984

[6] J. E. Mazo, "On the Independence Theory of Equalizer Convergence", Bell Sys. Tech. J. Vol.58, No.5, pp. 963-993, May-June 1979.

[7] K. M. Aleong, A Technique for Combining Equalization with Differential Detection M.Eng. Thesis, Dept. Elec. Eng., McGill Univ., Montreal, Quebec, Canada, June 1991.

[8] B. Widrow, M. E. Hoff Jr., "Adaptive Switch ing Circuits", IRE Wescon Conv., Pt. 4, pp. 96-104, 1960

[9] D. N. Godard, "Channel Equalization Using a Kalman Filter for Fast Data Transmission", IBM J. Res. Develop., Vol. 18, pp. 267-273, May 1974.

\begin{tabular}{|c|c|c|}
\hline & channel A & channel B \\
\hline $\begin{array}{l}\text { step-size } \\
\text { constellation } \\
\end{array}$ & $0.005 \quad 0.050$ & $0.005 \quad 0.050$ \\
\hline 8PSK & $0.026 \quad 0.032$ & $0.021 \quad 0.026$ \\
\hline $8 \mathrm{~V} 29$ & $0.025 \quad 0.029$ & $0.023 \quad 0.028$ \\
\hline 16QAM & $0.027 \quad 0.032$ & $0.023 \quad 0.028$ \\
\hline $16 \mathrm{~V} 29$ & $\begin{array}{ll}0.025 & 0.029 \\
\end{array}$ & $0.024 \quad 0.029$ \\
\hline MMSE & 0.0210 & 0.0176 \\
\hline
\end{tabular}

Table 1 : Comparison of residual MSE and MMSE for $\mathrm{SNR}=25 \mathrm{~dB}$. 


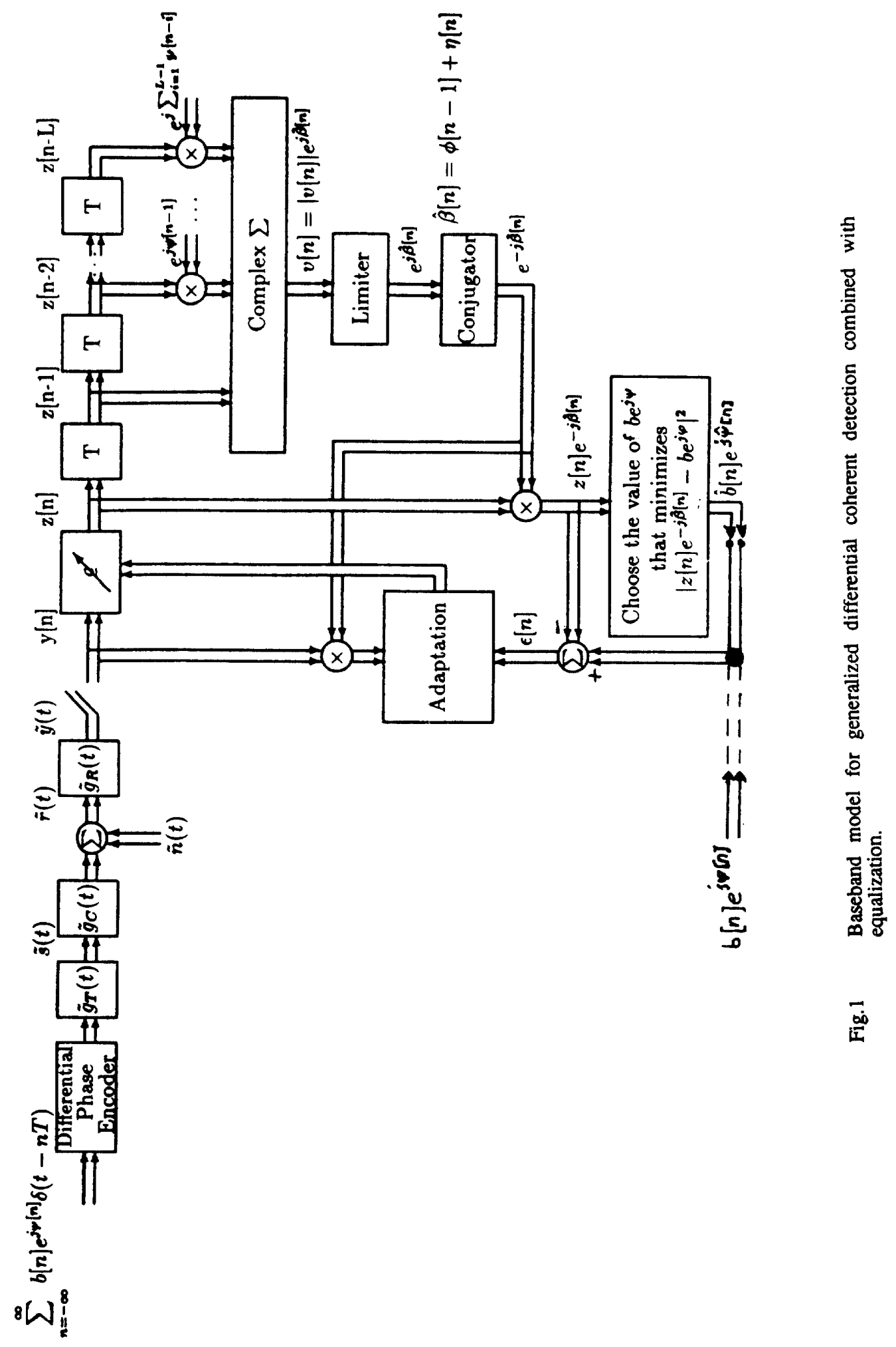




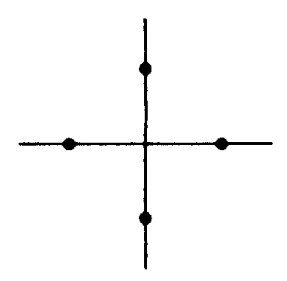

4PSK

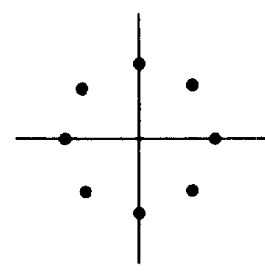

8PSK

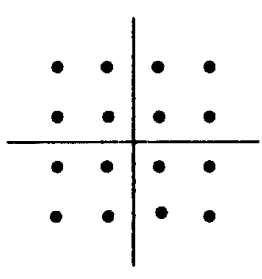

16QAM

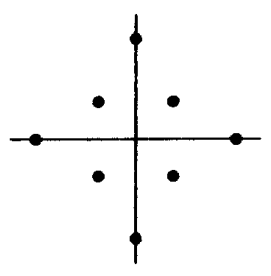

$8 \mathrm{~V} 29$

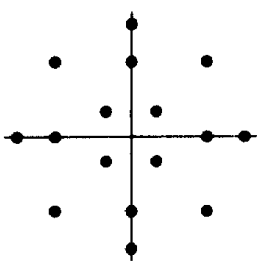

$16 \mathrm{~V} 29$

Fig.2 Two dimensional signal constellations considered in this work.

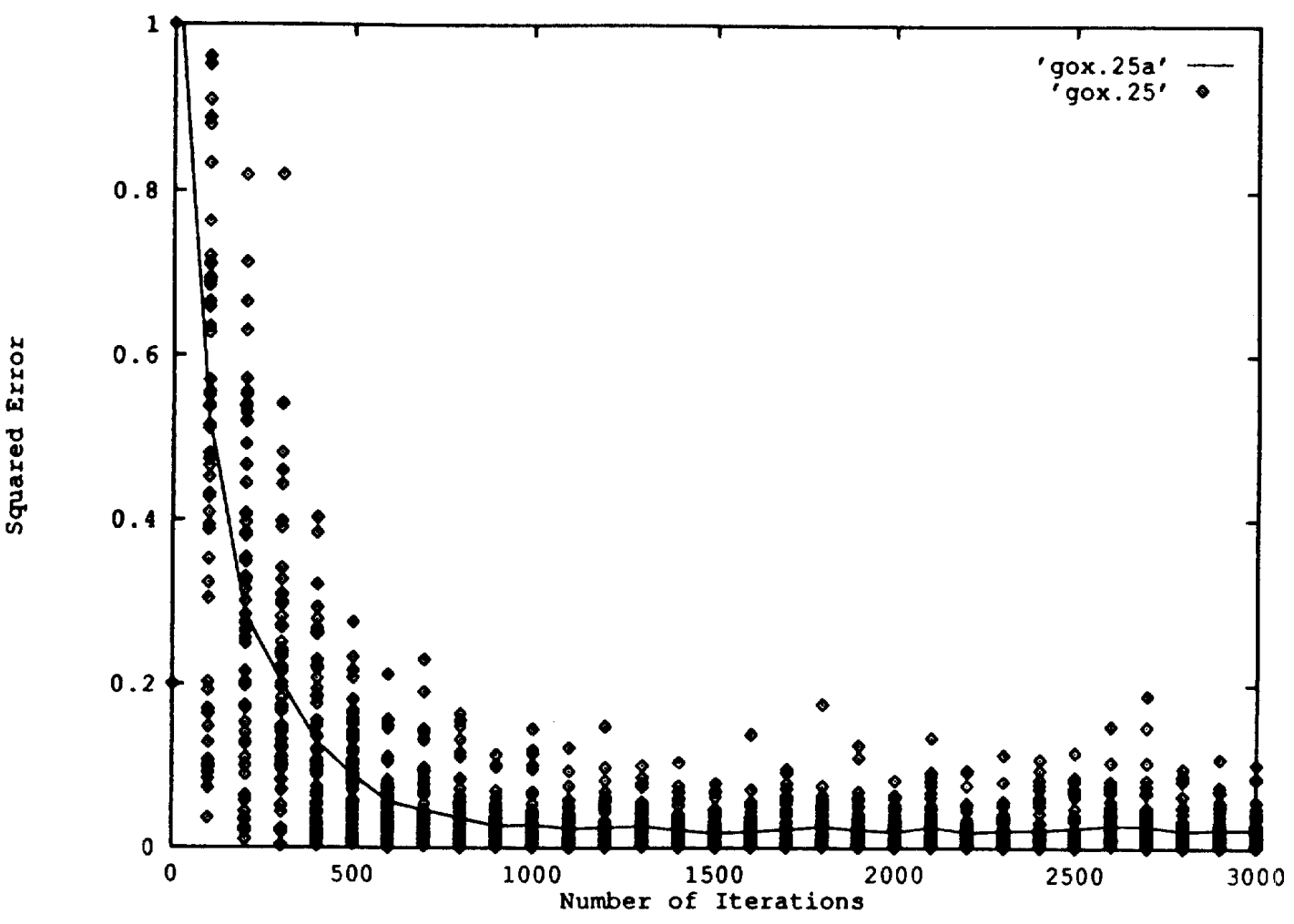

Figure 3 : Sixty trials and Average Learning Curve for 16QAM, channel $X$ and $\lambda=0.005$ 


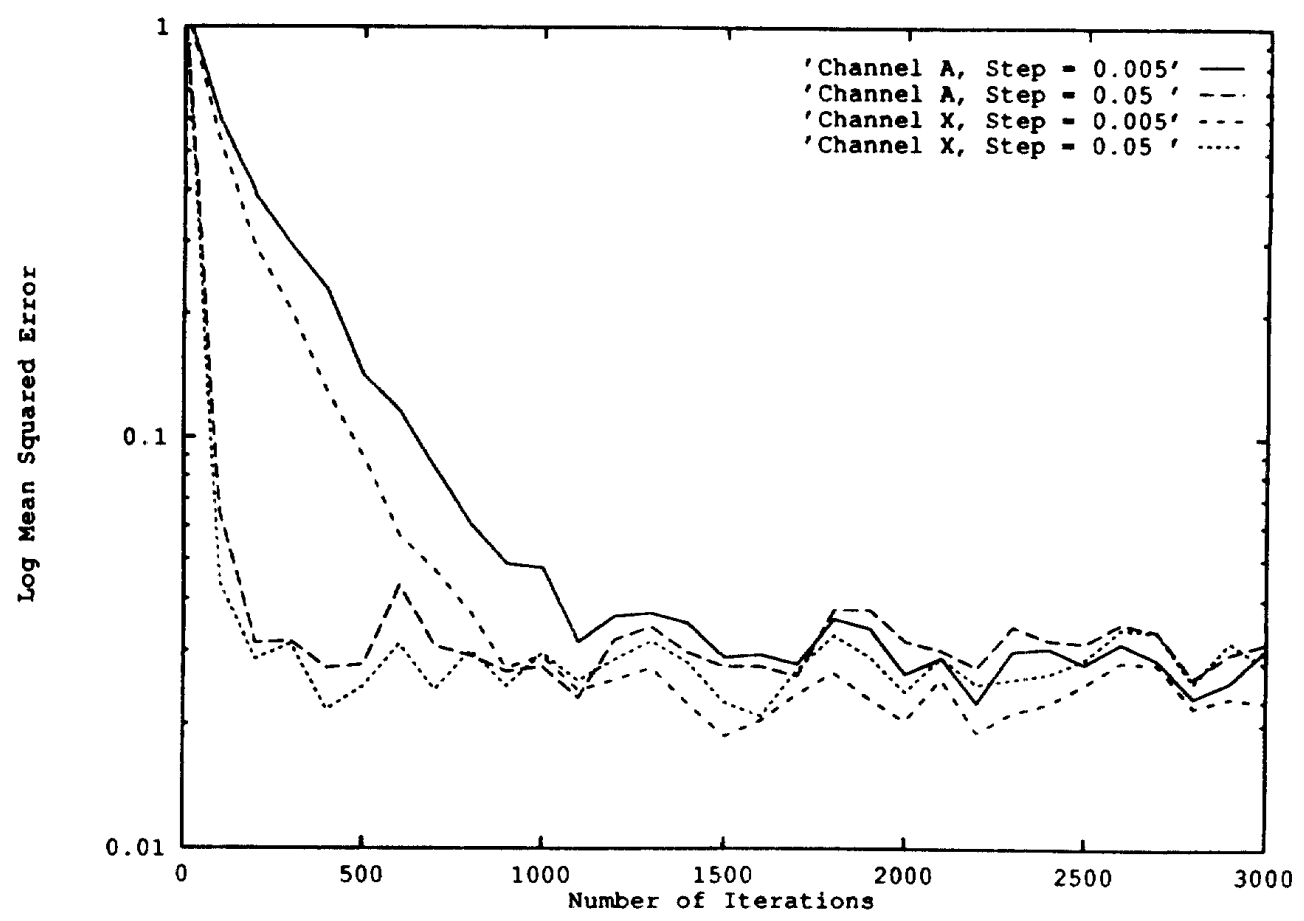

Figure 4 : Average Learning Curves for 16QAM.

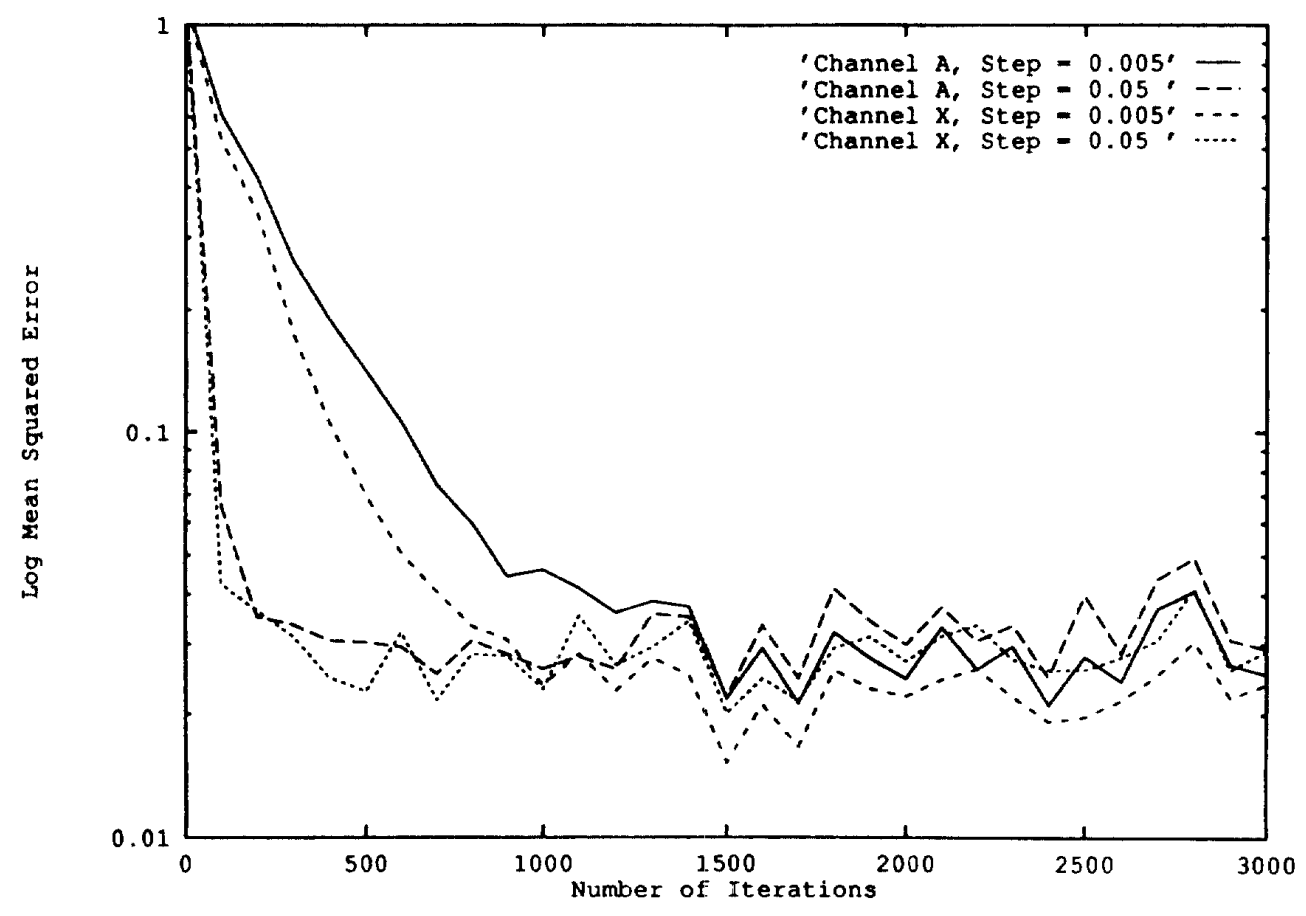

Figure 5: Average Learning Curves for 16V29. 